\title{
SIMULATION STUDY OF MULTI-STAGE PROTON CYCLOTRON RESONANCE ACCELERATOR - PROCRA*
}

\author{
Changbiao Wang ${ }^{1}$ and J. L. Hirshfield ${ }^{1,2}$ \\ ${ }^{1}$ Beam Physics Laboratory, Yale University, 272 Whitney Ave., New Haven, CT 06511, USA \\ ${ }^{2}$ Omega-P, Inc., 345 Whitney Ave., New Haven, CT 06511, USA
}

\begin{abstract}
Simulations are presented for a proton accelerator in which a pulsed proton beam is passed through a cascade of normal conducting cavities in a uniform magnetic field and accelerated using cyclotron resonance. To keep the accelerated proton beam in resonance with the rf fields, frequencies for successive cavities are lowered by a fixed frequency interval. It is shown, for an accelerator with a cascade of 8 cavities with $5 \mathrm{MHz}$ frequency intervals dropping from $100 \mathrm{MHz}$ at the first cavity in a $6.7 \mathrm{~T}$ magnetic field, that a $10 \mathrm{kV}-0.14 \mathrm{~A}$ injected proton beam is accelerated to $0.516 \mathrm{GeV}$, with an efficiency of $73 \%$ and an average acceleration gradient of over $19 \mathrm{MV} / \mathrm{m}$.
\end{abstract}

\section{INTRODUCTION}

High intensity proton accelerators have numerous promising applications, including the transmutation of nuclear wastes [1]. A novel high current, high gradient, high efficiency proton accelerator was earlier proposed [2] in which a pulsed proton beam is passed through a cascade of cavities immersed in a uniform magnetic field and accelerated using cyclotron resonance.

This proton cyclotron resonance accelerator (PROCRA) differs from the microwave cyclotron autoresonance accelerator (CARA) [3] and the laser-driven cyclotron auto-resonance accelerator (LACARA) [4] in at least three ways: (i) CARA and LACARA are microwavedriven and laser-driven electron accelerators, while
PROCRA would be a VHF-band protron machine [2], operating at frequencies in the range $40-120 \mathrm{MHz}$ for acceleration up to $0.5-1.0 \mathrm{GeV}$; (ii) CARA and LACARA employ non-uniform magnetic fields having spatial profiles designed to preserve cyclotron resonance along the acceleration path, while PROCRA employs a uniform magnetic field in which slippage in and out of resonance phase occurs in each stage; (iii) the electron beam in CARA and LACARA can in principle be continuous or pulsed, but the proton beam in PROCRA must be pulsed.

Since a uniform magnetic field in PROCRA is needed to avoid stalling and to obtain significant acceleration, successive cavity stages operate at successively lower $\mathrm{rf}$ frequencies to maintain near-resonance as the proton mass increases. With a fixed frequency difference $\Delta f$ between successive cavities, injection of protons must be at time intervals of $1 / \Delta f$ in order to preserve phase synchronism in all cavities. It was shown in the previous study [2] that the energy spread of accelerated protons increases with duty factor (ratio of proton bunch width to pulse period).

Based on previous work, this paper presents results of a computational study for a PROCARA having a cascade of 8 cavities, in which a $10 \mathrm{keV}-0.14 \mathrm{~A}$ proton beam is accelerated to more than $0.5 \mathrm{GeV}$. Simulations show this to be possible with an rf-to-beam power efficiency of $73 \%$, and an average acceleration gradient of $19.2 \mathrm{MV} / \mathrm{m}$.

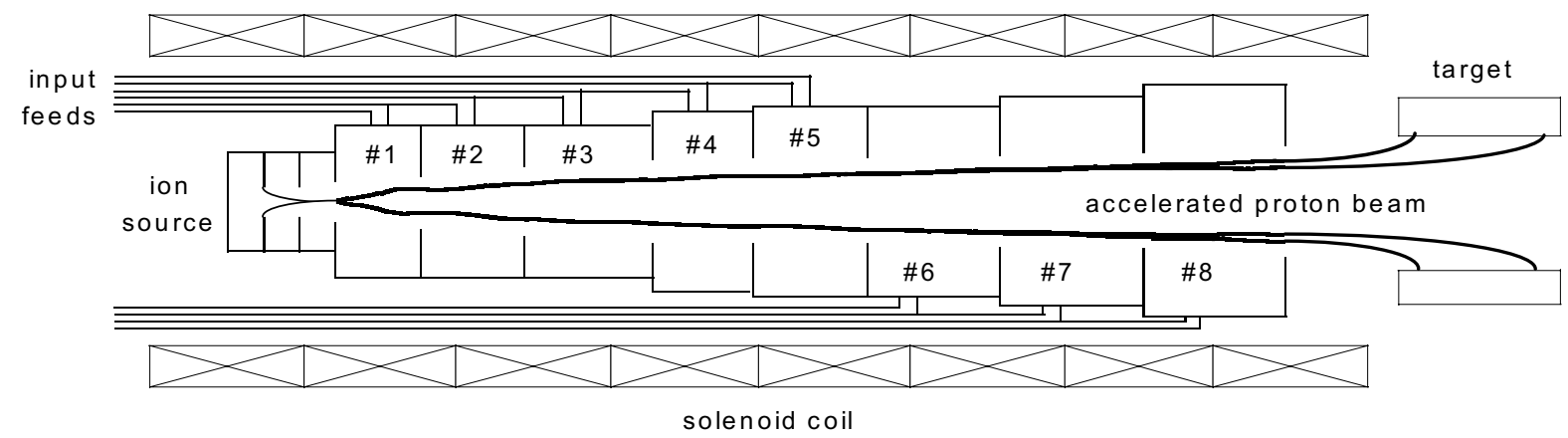

Figure 1: Sketch of 70-MW proton cyclotron resonance accelerator consisting of a cascade of 8 cavities, with a $5 \mathrm{MHz}$ frequency interval dropping down from $100 \mathrm{MHz}$ at the cavity \#1 to $65 \mathrm{MHz}$ at cavity \#8. Focused by a $6.7 \mathrm{~T}$ magnetic field produced by superconducting coils, a $10 \mathrm{keV}-0.14$ A proton beam from the ion source is accelerated to $>0.5 \mathrm{GeV}$ and is thereafter incident on a target. The envelope of gyrating protons shown is obtained from computations.

*Work supported by US DoE. 


\section{DESCRIPTION OF THE PROTON ACCELERATOR - PROCRA}

The PROCRA described here consists of a cascade of 8 cavities operating in rotating $T E_{111}$ modes, as shown in Fig. 1. Protons are injected at $10 \mathrm{keV}$ directly from an ion source and assumed to have zero emittance. The bunch width for the injected proton beam is $5 \mathrm{~ns}$ and the bunch period is $200 \mathrm{nsec}$, for a duty cycle of $2.5 \%$; the average current is $0.136 \mathrm{~A}$. The operating frequency is $100 \mathrm{MHz}$ for cavity \#1, $95 \mathrm{MHz}$ for cavity \#2, etc., with a constant frequency interval $\Delta f=-5 \mathrm{MHz}$ between all cavities. The magnetic field is $6.7 \mathrm{~T}$, rf drive power for each cavity is $12 \mathrm{MW}$, and intrinsic quality-factors $Q_{0}$ are $10^{5}$. Cavity frequencies $f$, radii $R$, lengths $L$, and loaded qualityfactors $Q_{L}$ for each stage are given in Table 1 .

Table 1: Parameters for each stage in the simulation.

Note: Radii for vaned cavities will be smaller [2].

\begin{tabular}{|c|c|c|c|c|c|}
\hline stage & $\begin{array}{c}f \\
(\mathrm{MHz})\end{array}$ & $\begin{array}{c}R \\
(\mathrm{~cm})\end{array}$ & $\begin{array}{c}L \\
(\mathrm{~cm})\end{array}$ & $Q_{L}$ & $\begin{array}{c}\text { final energy } \\
(\mathrm{MeV})\end{array}$ \\
\hline$\# 1$ & 100 & 110 & 249 & 30,000 & 61.70 \\
\hline$\# 2$ & 95 & 110 & 291 & 30,000 & 123.30 \\
\hline$\# 3$ & 90 & 110 & 361 & 24,800 & 189.46 \\
\hline$\# 4$ & 85 & 130 & 291 & 32,500 & 248.70 \\
\hline$\# 5$ & 80 & 137 & 313 & 38,000 & 303.54 \\
\hline$\# 6$ & 75 & 137 & 385 & 23,500 & 371.03 \\
\hline$\# 7$ & 70 & 150 & 391 & 18,500 & 442.41 \\
\hline$\# 8$ & 65 & 165 & 402 & 17,000 & 515.81 \\
\hline
\end{tabular}

To keep protons resonant with the rf fields, each cavity operating frequency should, in principle, be equal to the local proton gyration frequency. However, it has been found that a small deviation from resonance is necessary to avoid stalling, defined as $\beta_{z} \rightarrow 0$. At cavity \#1, adjustments in magnetic field effect the deviation. For subsequent cavities, the optimum phase is obtained by adjusting the phase of the input rf power.

In the simulations, several approximations are made. Because the proton gyration radius is considerably less than the cavity radius as shown in Fig. 1, distortions of rf field patterns at openings between cavities are assumed to be negligible. The energy increase per rf cycle as protons pass through the cavity is taken equal to the cavity stored energy multiplied by $2 \pi\left(1 / Q_{L}-1 / Q_{0}\right)<<1$, since rapid variations of field amplitude with time can be neglected.

The cavity fields for $\mathrm{TE}_{111}$ rotating modes are given by

$$
\begin{aligned}
& E_{r}=-\hat{B} \frac{\omega}{k_{c}} \frac{1}{k_{c} r} J_{1}\left(k_{c} r\right) \sin (\pi z / L) \sin (\omega t-\theta), \\
& E_{\theta}=\hat{B} \frac{\omega}{k_{c}} J_{1}^{\prime}\left(k_{c} r\right) \sin (\pi z / L) \cos (\omega t-\theta),
\end{aligned}
$$

where $\omega$ is the cavity operating frequency, $k_{c}=j_{11}^{\prime} / R$ with $j_{11}^{\prime}$ the first zero of the Bessel function derivative. Energy conservation is invoked to find the field amplitude $\hat{B}$ and average beam current $I$, namely,

$$
\begin{aligned}
& \hat{B}=\left[\frac{1}{\varepsilon_{0} \pi R^{2} L} \frac{k_{c}^{2}}{\omega^{3}} \frac{4 Q_{L} P_{i n}}{J_{1}^{2}\left(j_{11}^{\prime}\right)\left(1-1 / j_{11}^{\prime 2}\right)}\right]^{1 / 2}, \\
& I=\left(1-\frac{Q_{L}}{Q_{0}}\right) \frac{q_{p} P_{i n}}{m_{p} c^{2}\left(<\gamma_{f}>-1\right)},
\end{aligned}
$$

where $P_{i n}$ is the input rf power, $q_{p}$ is the proton charge, $m_{p}$ is the rest mass, and $\left\langle\gamma_{f}\right\rangle$ is the average relativistic energy factor at the end of the cavity.

The interaction of protons with rf fields is calculated stage-by-stage. When protons go into a given stage, the relative phase of the rf drive power is adjusted to maintain approximate resonance with the cavity fields; the proton current is then adjusted to the design value by specifying $Q_{L}$. At the optimum relative phase, minimum energy spread was found to accompany maximum final acceleration energy [2].

\section{SIMULATION RESULTS}

Results from a preliminary computational study are presented here to illustrate the new concept for proton acceleration. Eight macro-particles are taken, initially distributed uniformly in phase over $5 \mathrm{~ns}$, or half a period of the input rf power of the first cavity.

Figure 2 shows the dependence of average relativistic energy factor $\langle\gamma\rangle$ and normalized axial velocity $\left.<\beta_{z}\right\rangle$ on axial distance $z$ for the 8-stage PROCRA. It is seen that the proton beam is accelerated from $10 \mathrm{keV}$ $(<\gamma>=1.000011)$ to $0.516 \mathrm{GeV} \quad(<\gamma>=1.549734)$, with an average acceleration gradient of $19.2 \mathrm{MeV} / \mathrm{m}$ and a rf-to-beam power efficiency of $73 \%$. The resulting average beam power is $70 \mathrm{MW}$. The acceleration energy after each stage is given in Table 1.

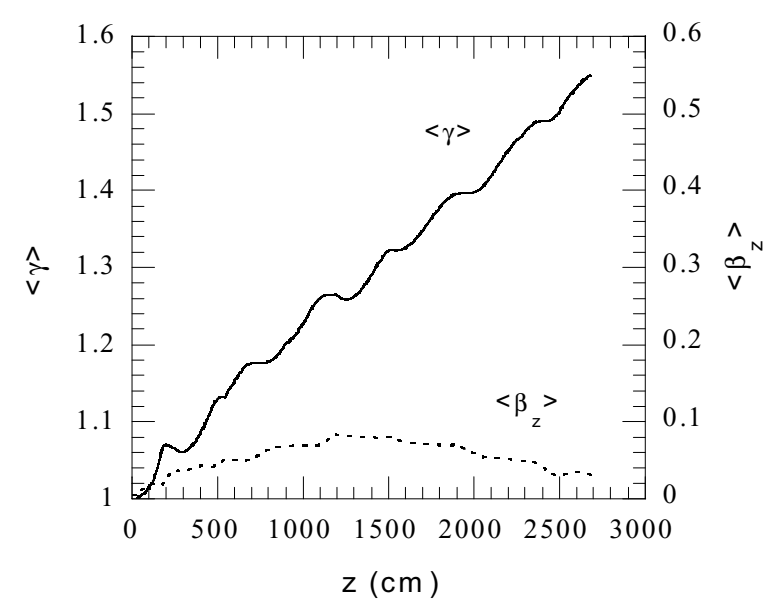

Figure 2: Dependence of average relativistic energy factor $\langle\gamma\rangle$ and normalized axial velocity $\left\langle\beta_{z}\right\rangle$ on axial distance $z$. Final proton energy is $515.8 \mathrm{MeV}$, beam current is $0.136 \mathrm{~A}$, average acceleration gradient is 19.2 $\mathrm{MeV} / \mathrm{m}$, and rf-to-beam efficiency is $73 \%$. 
From Fig. 2, it can be seen that the average axial velocity tends to increases in the first four stages and afterwards it begins to decrease, with a final velocity ratio of $\left\langle\beta_{\perp}\right\rangle\left|<\beta_{z}\right\rangle=24.2$ at $z=2684 \mathrm{~cm}$, corresponding to a pitch angle of $87.6^{\circ}$. The angle is decreased where the accelerated proton beam is incident on the target by gradually reducing the magnetic field. The beam is seen to sweep around the extended target, illuminating an area taken to be large enough for dissipation of its $70 \mathrm{MW}$.

It should be noted that there is an acceleration energy limit to classical CARA acceleration for a $T E_{111}$ mode cavity, wherein a resonant magnetic field is applied. The energy limit is given by $(\Delta E)_{\max }=E_{0} \sqrt{\left(\gamma_{0}+1\right) /\left(\gamma_{0}-1\right)}$, where $E_{0}$ is the initial particle energy, $\gamma_{0}$ is the initial relativistic energy factor, and $(\Delta E)_{\max }$ is the maximum energy gain. If the initial proton energy $E_{0}$ is $10 \mathrm{keV}$ with $\gamma_{0}-1=1.00661 \times 10^{-5}$, the maximum energy gain is 4.33 MeV. From Table 1, it can be seen that in the PROCRA with a uniform magnetic field the energy gain for the first stage is $61.69 \mathrm{MeV}$, much higher than the maximum energy gain in CARA. This can be understood since, in the CARA configuration, there is a transverse magnetic field component associated with the gradient in axial magnetic field; this transverse field increases with acceleration energy and thus decreases the axial velocity, thereby leading to stalling. In PROCRA (unlike CARA and LACARA where electron axial velocities are relativistic) initial proton axial velocities are so small (see Fig. 2) that stalling can occur before protons achieve much acceleration if a resonant magnetic field were to be applied. Stalling can be avoided in a uniform or axiallydecreasing guide magnetic field.

Figures 3 and 4 show projections in the transverse $(x-y)$ and longitudinal $(x-z)$ planes of the orbit of a single proton during the course of its acceleration. Each proton executes 167 turns as it traverses all eight cavities. In the

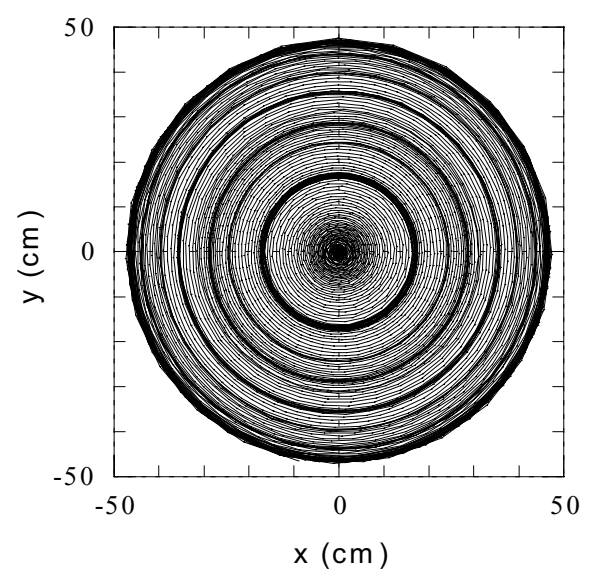

Figure 3: Projection in the transverse plane of the orbit of a proton undergoing acceleration. The gyration radius increases with acceleration energy. first cavity, the proton gyration radius grows from zero and executes the greatest number of turns. It is seen from Fig. 2 that the proton acceleration energy and gyration radius change slowly or even decrease slightly near cavity ends due to phase slippage; this explains the apparent crowding of orbits in Fig. 3.

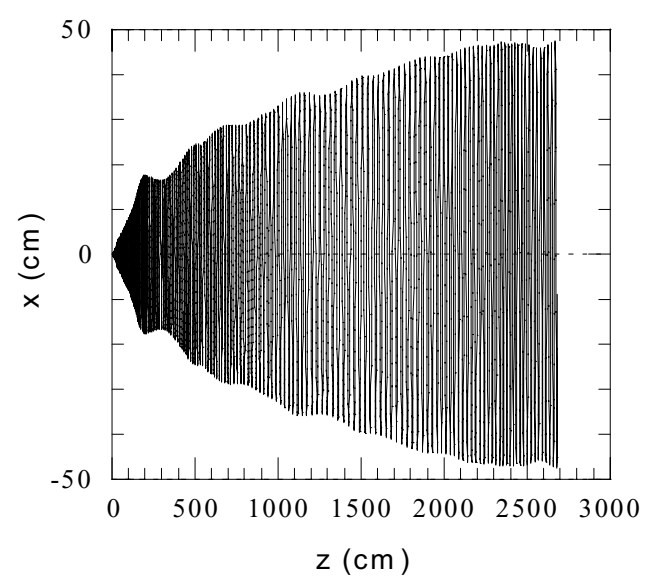

Figure 4: Projection in the longitudinal plane of the orbit of a proton undergoing acceleration. Each proton executes 167 turns during acceleration in this example.

\section{REMARKS}

Room temperature proton linear accelerators usually have acceleration gradients of the order of $2 \mathrm{MeV} / \mathrm{m}$. For applications requiring proton energies in the range $0.5-2$ $\mathrm{GeV}$, this leads to machine lengths of $0.25-1 \mathrm{~km}$, a length that carries a significant real estate cost and that can preclude some applications. The PROCRA concept presented here may allow reduction by a factor-of-ten in the length. This could allow the installation of proton accelerators for some applications that may now be precluded on account of space limitations.

\section{REFERENCES}

[1] W. T. Weng, "Ultra-high intensity proton accelerators and their applications," in Proc. of the 1997 Particle Accelerator Conference, Vol. 1, pp. 42-46, (IEEE, 1998).

[2] J. L. Hirshfield, C. Wang, and R. Symons, "Multistage, high-gradient, cyclotron resonance proton accelerator concept," AAC 2000.

[3] C. Wang and J. L. Hirshfield, "Energy limit in cyclotron autoresonance acceleration," Phys. Rev. E 51, 2456 (1995).

[4] J. L. Hirshfield and C. Wang, "Laser-driven electron cyclotron autoresonance accelerator with production of an optically chopped electron beam," Phys. Rev. E 61,7252 (2000). 